\title{
Positron-molecule annihilation by capture into vibrational Feshbach resonances of infrared-active modes
}

\author{
G. F. Gribakin* and C. M. R. Lee甘 \\ Department of Applied Mathematics and Theoretical Physics, \\ Queen's University, Belfast BT7 1NN, Northern Ireland, UK
}

\begin{abstract}
Enhanced positron annihilation on polyatomic molecules is a long-standing and complex problem. We report the results of calculations of resonant positron annihilation on methyl halides. A free parameter of our theory is the positron binding energy. A comparison with energy-resolved annihilation rates measured for $\mathrm{CH}_{3} \mathrm{~F}, \mathrm{CH}_{3} \mathrm{Cl}, \mathrm{CH}_{3} \mathrm{Br}$ [Barnes et al. Phys. Rev. A 74, 012706 (2006)] shows good agreement and yields estimates of the binding energies.

PACS numbers: $34.85 .+\mathrm{x}, 78.70 . \mathrm{Bj}, 34.50 . \mathrm{Ez}, 36.20 . \mathrm{Ng}$
\end{abstract}

In this paper we calculate the positron-molecule annihilation rate due to resonant capture of positrons by infrared-active vibrational modes, and observe good agreement with recent experimental data for methyl halides 1 .

When a fast positron interacts with matter, it undergoes a quick succession of ionizing and other inelastic collisions, and slows down to $\mathrm{eV}$ or thermal energies before annihilation. The low-energy positron annihilation rate in a gas with number density $n$ is usually parametrized as

$$
\lambda \equiv \sigma_{a} v n=\pi r_{0}^{2} c n Z_{\mathrm{eff}},
$$

where $\sigma_{a}$ is the annihilation cross section, $v$ is the positron velocity, $c$ is the speed of light, $r_{0}$ is the classical electron radius, and $Z_{\text {eff }}$ is an effective number of electrons per gas atom or molecule, that contribute to annihilation [2, 3]. Originally, $Z_{\text {eff }}$ was introduced in expectation that the annihilation rate would be in proportion to the number of target electrons, $Z$. However, early experiments 4, 5, [6] and later systematic studies 7, 8, [9] found that for many polyatomic molecules $Z_{\text {eff }}$ exceeded $Z$ by orders of magnitude. It also showed strong chemical sensitivity and rapid growth with molecular size (see review [10]).

Explanations of high molecular $Z_{\text {eff }}$ were sought in terms of positron virtual or weakly bound states 11], resonances [12, 13], long-lived vibrationally excited positron-molecule complexes [8], and virtual Ps formation 14. At the same time, annihilation calculations which neglected molecular vibrations, failed to reproduce "anomalous" $Z_{\text {eff }}$ for polyatomics [15, 16], but gave evidence that $Z_{\text {eff }}$ depend on the molecular geometry [17].

These efforts highlight the fact that positron-molecule annihilation is a complex problem. Nevertheless, a theory developed in Refs. [18, 19, 20] provides a framework for analyzing this phenomenon. There are two basic mechanisms of positron annihilation, direct and resonant. The direct mechanism applies to both atoms and molecules and involves annihilation of an incident positron "in flight". Its contribution is enhanced when a low-lying virtual or weakly bound positron state is present, leading to $Z_{\text {eff }}$ up to $10^{3}$ for room-temperature positrons 18, 19.

Resonant annihilation occurs for molecules capable of binding the positron. To be captured into a bound state, the positron energy must be absorbed by a vibrational excitation of the positron-molecule complex. This gives rise to a vibrational Feshbach resonance (VFR) at the incident positron energy $\varepsilon=E_{\nu}+\varepsilon_{0}$, where $E_{\nu}$ is the vibrational excitation energy, and $\varepsilon_{0}<0$ is the positron bound state energy. The positron bound in the VFR can annihilate (or undergo detachment). The probability of annihilation is proportional to the resonance lifetime.

For non-monoenergetic positrons and closely spaced resonances, their contribution to $Z_{\text {eff }}$ is proportional to the vibrational level density at $E \approx \varepsilon-\varepsilon_{0}[18,19]$. If the positron VFR were due to excitation of fundamentals alone, this density would be proportional to the number of modes. Experimental $Z_{\text {eff }}$ show much faster increase (e.g., $Z_{\text {eff }}=3500,11300$, and 37800 for $\mathrm{C}_{3} \mathrm{H}_{8}, \mathrm{C}_{4} \mathrm{H}_{10}$, and $\mathrm{C}_{5} \mathrm{H}_{12}$, respectively). This means that positron attachment involves excitation of overtones and combination vibrations. Large $Z_{\text {eff }}$ are then related to high total vibrational spectrum densities in the polyatomics.

The important role of vibrations was recently verified by measuring the energy dependence of $Z_{\text {eff }}$ at sub-eV energies with a high-resolution positron beam 21, 22]. These experiments uncovered peaks in $Z_{\text {eff }}$, whose energies corresponded to those of molecular vibrational modes. In particular, for all alkanes larger than methane, $Z_{\text {eff }}$ displayed a prominent $\mathrm{C}-\mathrm{H}$ maximum. Its downshift from the $\mathrm{C}-\mathrm{H}$ mode energy $(0.37 \mathrm{eV})$ provided a measure of the positron binding energy 23]. Observation of such peaks means that excited fundamentals act as vibrational doorway states 20], leading to multimode vibrations through intramolecular vibrational relaxation (IVR).

Therefore, to compute $Z_{\text {eff }}$ for polyatomics, one must account for strong electron-positron correlations and positron binding, the interaction between positronic and vibrational degrees of freedom and intramolecular vibra- 
tional mixing. This makes ab initio calculations of high molecular $Z_{\text {eff }}$ very difficult. However, as we show below, for small polyatomics some basic features of resonant annihilation can be tested by relatively simple calculations.

The resonant part of the annihilation cross section can be written using the Breit-Wigner formalism 19, 20, 24],

$$
\sigma_{a}=\frac{\pi}{k^{2}} \sum_{\nu} \frac{g_{\nu} \Gamma_{\nu}^{a} \Gamma_{\nu}^{e}}{\left(\varepsilon-E_{\nu}-\varepsilon_{0}\right)^{2}+\Gamma_{\nu}^{2} / 4}
$$

where $\Gamma_{\nu}^{a}, \Gamma_{\nu}^{e}$, and $\Gamma_{\nu}$ are the annihilation, elastic and total widths of $\nu$ th resonance, $g_{\nu}$ is its degeneracy, and $k$ is the positron momentum (atomic units are used). The annihilation width is proportional to the electron density at the positron in the positron bound state, $\rho_{e p}$,

$$
\Gamma_{\nu}^{a}=\pi r_{0}^{2} c \rho_{e p} .
$$

From Eqs. (11) and (21), the resonant $Z_{\text {eff }}$ is given by

$$
Z_{\mathrm{eff}}^{(\mathrm{res})}=\frac{\pi}{k} \rho_{e p} \sum_{\nu} \frac{g_{\nu} \Gamma_{\nu}^{e}}{\left(\varepsilon-E_{\nu}-\varepsilon_{0}\right)^{2}+\Gamma_{\nu}^{2} / 4} .
$$

We will now use this equation to calculate the contribution of infrared-active modes to $Z_{\text {eff }}^{\text {(res) }}$.

Consider a compact polyatomic molecule that can bind the positron with a small binding energy $\left|\varepsilon_{0}\right| \equiv \kappa^{2} / 2 \ll 1$ $\mathrm{eV}$. The wavefunction of the bound positron is very diffuse and behaves as $\varphi_{0}=A r^{-1} e^{-\kappa r}$ outside the molecule. Since large distances dominate, the normalization constant is given by $A \simeq(\kappa / 2 \pi)^{1 / 2}[25]$.

Suppose that the vibrational modes in this small-sized polyatomic are not mixed with overtones or combination vibrations. Given the smallness of the binding energy, the vibrational excitation energies of the positron-molecule complex should be close to the fundamental frequencies $\omega_{\nu}$ of the neutral molecule. In this case the sum in Eq. (44) is over the modes $\nu$, and $E_{\nu} \approx \omega_{\nu}$. Some (or even all) of these modes can be infrared active. The positron capture into such excited states is mediated by the longrange dipole coupling. This allows one to calculate their contribution to $Z_{\text {eff }}^{\text {(res) }}$.

Consider a positron with momentum $\mathbf{k}$ incident on the molecule in the vibrational ground state $\Phi_{0}(\mathbf{R})$, where $\mathbf{R}$ represents all the molecular coordinates. If $k^{2} / 2 \approx$ $\omega_{\nu}+\varepsilon_{0}$, the positron can be captured in the VFR, where it is bound to the molecule in a vibrationally excited state $\Phi_{\nu}(\mathbf{R})$. The corresponding width $\Gamma_{\nu}^{e}$ can be found from

$$
\Gamma_{\nu}^{e}=2 \pi \int\left|A_{\nu \mathbf{k}}\right|^{2} \delta\left(k^{2} / 2-\omega_{\nu}-\varepsilon_{0}\right) \frac{d^{3} k}{(2 \pi)^{3}},
$$

where $A_{\nu \mathbf{k}}$ is the capture amplitude. We calculate it by using a method similar to the Born-dipole approximation
[26, 27], as

$$
\begin{aligned}
A_{\nu \mathbf{k}} & =\int \varphi_{0}(\mathbf{r}) \Phi_{\nu}^{*}(\mathbf{R}) \frac{\hat{\mathbf{d}} \cdot \mathbf{r}}{r^{3}} e^{i \mathbf{k} \cdot \mathbf{r}} \Phi_{0}(\mathbf{R}) d \mathbf{r} d \mathbf{R} \\
& =\frac{4 \pi i}{3} \frac{\mathbf{d}_{\nu} \cdot \mathbf{k}}{\sqrt{2 \pi \kappa}}{ }_{2} F_{1}\left(\frac{1}{2}, 1 ; \frac{5}{2} ;-\frac{k^{2}}{\kappa^{2}}\right),
\end{aligned}
$$

where $\hat{\mathbf{d}}$ is the dipole moment operator for the molecule, $\mathbf{d}_{\nu}=\left\langle\Phi_{\nu}|\hat{\mathbf{d}}| \Phi_{0}\right\rangle$, and ${ }_{2} F_{1}$ is the hypergeometric function [28]. Substitution of Eq. (6) into Eq. (5) gives

$$
\Gamma_{\nu}^{e}=\frac{16 \omega_{\nu} d_{\nu}^{2}}{27} h(\xi)
$$

where $h(\xi)=\xi^{3 / 2}(1-\xi)^{-1 / 2}\left[{ }_{2} F_{1}\left(\frac{1}{2}, 1 ; \frac{5}{2} ;-\xi /(1-\xi)\right)\right]^{2}$ is a dimensionless function of $\xi=1+\varepsilon_{0} / \omega_{\nu}$, such that $\xi(0)=\xi(1)=0$, and $h_{\max } \approx 0.75$ at $\xi \approx 0.89$.

Equation (7) shows that the elastic width of a positron VFR for an infrared active mode is basically determined by its frequency $\omega_{\nu}$ and transition dipole amplitude $d_{\nu}$, known from infrared absorption measurements [29].

For weakly bound positron states the density $\rho_{e p}$ is a linear function of $\kappa[19]$. It can be estimated as

$$
\rho_{e p}=(F / 2 \pi) \kappa,
$$

with $F \approx 0.66[19]$. The same constant characterizes the contribution of direct annihilation, $Z_{\mathrm{eff}}^{(\mathrm{dir})} \simeq F /\left(\kappa^{2}+k^{2}\right)$ 19]. It is enhanced at small positron momenta by the presence of a weakly-bound (or virtual) state [11, 30].

In a recent paper [1] measurements of $Z_{\text {eff }}$ for $\mathrm{CH}_{3} \mathrm{Cl}$ and $\mathrm{CH}_{3} \mathrm{Br}$ using a cold trap-based positron beam, have been reported. The energy dependence of $Z_{\text {eff }}$ for these molecules (and $\mathrm{CH}_{3} \mathrm{~F}$ measured earlier [22]) shows peaks close to the vibrational mode energies. This points to an important contribution of resonant annihilation in all three molecules, although the maximum $Z_{\text {eff }}$ value for $\mathrm{CH}_{3} \mathrm{~F}$ (250) is much lower than those for $\mathrm{CH}_{3} \mathrm{Cl}$ and $\mathrm{CH}_{3} \mathrm{Br}$ (1600 and 2000, respectively).

These molecules have $C_{3 v}$ symmetry, and all six of their vibrational modes are infrared active (see Table【for $\mathrm{CH}_{3} \mathrm{Cl}$ ). Methyl halides are also relatively small, which means that IVR may not take place 31. This makes them ideal for application of our theory. Equations (4), (77), and (8) allow one to calculate the contribution of all VFR to $Z_{\text {eff }}^{\text {(res) }}$, and the only free parameter of the theory, i.e., the positron binding energy, can be chosen by comparison with experimental $Z_{\text {eff }}$.

In order to do this, $Z_{\text {eff }}^{(\text {res })}$ from Eq. (4) must be averaged over the energy distribution of the positron beam 22]. The latter can be modelled by a combination of the Gaussian distribution in the longitudinal direction $(z)$ and Maxwellian distribution in the transversal direction $(\perp)$. The corresponding probability density of the total positron energy, $\varepsilon_{\perp}+\varepsilon_{z}$, is

$$
f_{\epsilon}\left(\varepsilon_{\perp}, \varepsilon_{z}\right)=\frac{1}{k_{B} T_{\perp} \sqrt{2 \pi \sigma^{2}}} \exp \left[-\frac{\varepsilon_{\perp}}{k_{B} T_{\perp}}-\frac{\left(\varepsilon_{z}-\epsilon\right)^{2}}{2 \sigma^{2}}\right],
$$


TABLE I: Characteristics of the vibrational modes of $\mathrm{CH}_{3} \mathrm{Cl}$.

\begin{tabular}{cccrcc}
\hline \hline Mode & Symmetry & $g_{\nu}$ & $\begin{array}{c}\omega_{\nu}{ }^{a} \\
(\mathrm{meV})\end{array}$ & $\begin{array}{c}d_{\nu} \\
\text { (a.u. })\end{array}$ & $\begin{array}{c}\omega_{\nu} d_{\nu}^{2} \\
\text { (a.u. })\end{array}$ \\
\hline$\nu_{1}$ & $\mathrm{a}_{1}$ & 1 & 363 & 0.0191 & $4.87 \times 10^{-6}$ \\
$\nu_{2}$ & $\mathrm{a}_{1}$ & 1 & 168 & 0.0176 & $1.91 \times 10^{-6}$ \\
$\nu_{3}$ & $\mathrm{a}_{1}$ & 1 & 91 & 0.0442 & $6.52 \times 10^{-6}$ \\
$\nu_{4}$ & $\mathrm{e}$ & 2 & 373 & 0.0099 & $1.34 \times 10^{-6}$ \\
$\nu_{5}$ & $\mathrm{e}$ & 2 & 180 & 0.0162 & $1.74 \times 10^{-6}$ \\
$\nu_{6}$ & $\mathrm{e}$ & 2 & 126 & 0.0111 & $5.66 \times 10^{-7}$ \\
\hline \hline
\end{tabular}

${ }^{a}$ Mode energies $\omega_{\nu}$ and dipole amplitudes $d_{\nu}$ from Ref. [29].

where $k_{B}$ is the Boltzmann constant, $T_{\perp}$ is an effective transversal temperature of the beam, $\epsilon$ is the mean longitudinal energy of the positrons, as measured by the retarding potential analyzer, and $\sigma=\delta_{z} / \sqrt{8 \ln 2}, \delta_{z}$ being the full width at half-maximum. The values of $k_{B} T_{\perp}$ and $\delta_{z}$ are taken from experiment to be $25 \mathrm{meV}$.

The averaging, $\bar{Z}_{\text {eff }}^{(\text {res })}(\epsilon)=\int Z_{\text {eff }}^{(\text {res })} f_{\epsilon}\left(\varepsilon_{\perp}, \varepsilon_{z}\right) d \varepsilon_{\perp} d \varepsilon_{z}$, can be done analytically, since the widths of the resonances, $\Gamma_{\nu}=\Gamma_{\nu}^{e}+\Gamma_{\nu}^{a}$, are small compared to the energy spread of the positron beam. Indeed, values from the last column of Table $\llbracket$ show that the elastic widths of the VFR are less then $0.1 \mathrm{meV}$. Typical annihilation widths are even smaller. For example, for a binding energy of $10 \mathrm{meV}$ ( $\kappa=0.027$ a.u.), Eqs. (3) and (8) yield $\Gamma_{\nu}^{a}=3 \times 10^{-9}$ a.u. $=0.1 \mu \mathrm{eV}$. These estimates also show that $\Gamma_{\nu} \approx \Gamma_{\nu}^{e}$, i.e., that the total width of the resonance is dominated by its elastic width.

Hence, to integrate over $\varepsilon_{\perp}$ and $\varepsilon_{z}$ we replace the BreitWigner profiles in Eq. (4) by $\delta$-functions, and obtain

$$
\bar{Z}_{\mathrm{eff}}^{(\mathrm{res})}(\epsilon)=2 \pi^{2} \rho_{e p} \sum_{\nu} \frac{g_{\nu} \Gamma_{\nu}^{e}}{k_{\nu} \Gamma_{\nu}} \Delta\left(\epsilon-\varepsilon_{\nu}\right)
$$

where $\varepsilon_{\nu}=k_{\nu}^{2} / 2=\omega_{\nu}+\varepsilon_{0}$ is the resonance energy, and

$$
\begin{aligned}
\Delta(E) & =\frac{1}{k_{B} T_{\perp}} \exp \left[\frac{\sigma^{2}}{2\left(k_{B} T_{\perp}\right)^{2}}\right] \exp \left(\frac{E}{k_{B} T_{\perp}}\right) \\
& \times\left\{1+\Phi\left[-\frac{1}{\sqrt{2}}\left(\frac{E}{\sigma}+\frac{\sigma}{k_{B} T_{\perp}}\right)\right]\right\},
\end{aligned}
$$

with $\Phi(x)$ being the standard error function.

The function $\Delta(E)$ is a convolution of the $\delta$-function with the positron energy distribution. It describes the appearance of a narrow resonance when measured with the trap-based positron beam, and is shown in Fig. 1 Due to the transversal energy component, its maximum is downshifted by $12 \mathrm{meV}$ from the true resonance position. The shape of $\Delta(E)$ is also markedly asymmetric, with an extended low energy tail. It agrees well with those of the observed $\mathrm{C}-\mathrm{H}$ peaks $[21,22]$. Note that the positron energy distribution was taken into account in experiment by assuming a $16 \mathrm{meV}$ difference between the positron total and longitudinal energies 22.

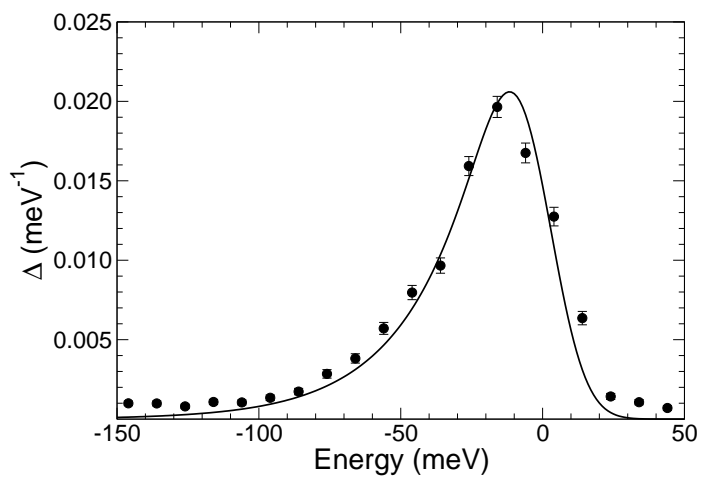

FIG. 1: Comparison of the resonance shape function $\Delta(E)$, for $k_{B} T_{\perp}=\delta_{z}=25 \mathrm{meV}$ (curve) with the measured $\mathrm{C}-\mathrm{H}$ peak in propane (circles) 21, 22]. For comparison, experimental $Z_{\text {eff }}$ has been scaled vertically and shifted horizontally.

In Fig. 2 we compare the beam-energy-averaged theoretical $Z_{\text {eff }}$ from Eq. (9) added to the direct contribution $Z_{\text {eff }}^{(\text {dir) }}$, with measured $Z_{\text {eff }}$ for methyl halides [1, 22]. Theoretical curves have been obtained using the binding energy of $\left|\varepsilon_{0}\right|=0.3,25$, and $40 \mathrm{meV}$, for $\mathrm{CH}_{3} \mathrm{~F}, \mathrm{CH}_{3} \mathrm{Cl}$ and $\mathrm{CH}_{3} \mathrm{Br}$, respectively.

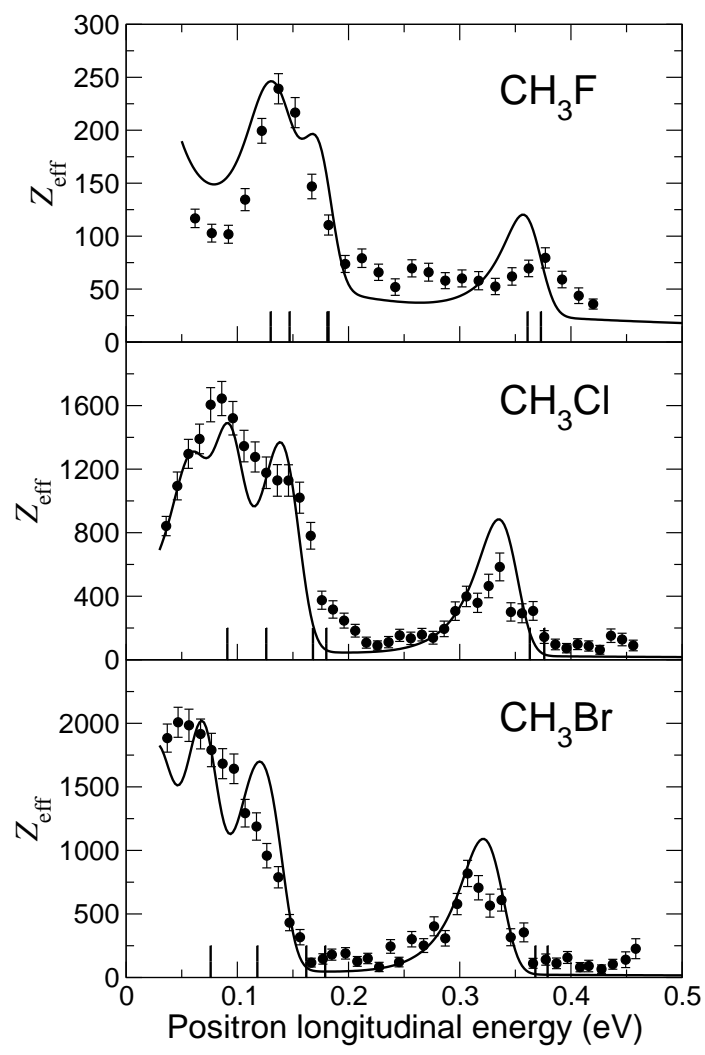

FIG. 2: Comparison between experimental $Z_{\text {eff }}(\bullet$, Ref. [1]) and theoretical $Z_{\text {eff }}$ obtained as a sum of the direct and resonant contributions, using the binding energy $\left|\varepsilon_{0}\right|=0.3 \mathrm{meV}$ $\left(\mathrm{CH}_{3} \mathrm{~F}\right), 25 \mathrm{meV}\left(\mathrm{CH}_{3} \mathrm{Cl}\right)$, and $40 \mathrm{meV}\left(\mathrm{CH}_{3} \mathrm{Br}\right)$. Vertical bars show the energies of molecular fundamentals. 
Given the complexity of the problem and the fact that $\varepsilon_{0}$ is the only free parameter in the calculation, the agreement between theory and experiment in Fig. 2] is remarkable. In accord with Eq. (91), every vibrational mode gives rise to a VFR, whose relative magnitude is determined by the factor $g_{\nu} / k_{\nu}$ (since $\left.\Gamma_{\nu}^{e} / \Gamma_{\nu} \approx 1\right)$. On the positron longitudinal energy scale, the resonances are downshifted from the mode energies by the positron binding energy and a further $12 \mathrm{meV}$ due to the positron energy distribution.

Besides determining the resonance positions, the binding energy also affects the overall magnitude of $Z_{\text {eff }}^{\text {(res) }}$ via $\rho_{e p} \propto\left|\varepsilon_{0}\right|^{1 / 2}$ [Eq. (8)]. Hence, the smallness of $Z_{\text {eff }}$ in $\mathrm{CH}_{3} \mathrm{~F}$ in comparison with those of $\mathrm{CH}_{3} \mathrm{Cl}$ and $\mathrm{CH}_{3} \mathrm{Br}$ is related to the weakness of its binding. This is in turn related to the smaller dipole polarizability and higher ionization potential of fluoromethane, which make it less attractive for the positron.

Note that the infrared absorption strengths of the modes and the corresponding elastic widths, $\Gamma_{\nu}^{e} \sim \omega_{\nu} d_{\nu}^{2}$, may vary considerably from mode to mode. On the other hand, the contribution of different modes to $Z_{\text {eff }}$ are similar, apart from energy shift and $g_{\nu} / k_{\nu}$ factor. As a result, the energy dependence of $Z_{\text {eff }}$ has little resemblance to the molecular infrared absorption spectra 1]. The relation $\Gamma_{\nu} \approx \Gamma_{\nu}^{e}$ also means that the contributions of the VFR are not sensitive to the exact values of the elastic widths. Therefore, our use of the "Born-dipole" approximation in the derivation of Eq. (7) is not expected to lead to sizeable errors in $Z_{\text {eff }}^{\text {(res) }}$.

In conclusion, we have presented a theory of positron annihilation by capture into vibrational resonances of infrared-active modes. It agrees well with measured $Z_{\text {eff }}$ for methyl halides and yields estimates of the positron binding energies for these molecules.

This theory can also be used to investigate the contribution of infrared-active-mode VFRs to $Z_{\text {eff }}$ in other small polyatomics that can bind positrons. Such calculations will likely underestimate the $Z_{\text {eff }}$, because the resonances associated with other (non-dipole) modes may contribute just as much, as long as their elastic widths are greater than the annihilation width.

In molecules where multiquantum vibrations are coupled by anharmonicity, the number of VFRs populated by positron capture will be greatly increased, leading to much higher $Z_{\text {eff }}$. However, the same coupling will also allow the VFR to decay by positron emission to vibrationally excited states of the molecule. This will increase the total resonance widths, thereby reducing their individual contributions. Calculation of $Z_{\text {eff }}$ for molecules with IVR is the next big challenge for the theory.

The authors are grateful to C. M. Surko and J. A. Young for a most helpful discussion of the positron energy distribution and valuable comments, and to A. V. Korol for discussions.
* E-mail address: g.gribakin@am.qub.ac.uk

† E-mail address: c.lee@qub.ac.uk

[1] L. D. Barnes, J. A. Young and C. M. Surko, Phys. Rev. A 74, 012706 (2006).

[2] P. A. Fraser, Adv. At. Mol. Phys. 4, 63 (1968).

[3] Here $\pi r_{0}^{2} c / v$ is the spin-averaged Born cross section of electron-positron annihilation, V. B. Berestetskii, E. M. Lifshitz, and L. P. Pitaevskii, Quantum electrodynamics (Pergamon, Oxford, 1982).

[4] M. Deutsch, Phys. Rev. 83, 866 (1951).

[5] D. A. L. Paul and L. Saint-Pierre, Phys. Rev. Lett. 11, 493 (1963).

[6] S. J. Tao, Phys. Rev. Lett. 14, 935 (1965).

[7] G. R. Heyland et al. Can. J. Phys. 60, 503 (1982).

[8] C. M. Surko et al. Phys. Rev. Lett. 61, 1831 (1988); T. J. Murphy and C. M. Surko, ibid. 67, 2954 (1991).

[9] K. Iwata et al. Phys. Rev. A 51, 473 (1995).

[10] C. M. Surko, G. F. Gribakin, and S. J. Buckman, J. Phys. B 38, R57 (2005).

[11] V. I. Goldanskii and Yu. S. Sayasov, Phys. Lett. 13, 300 (1964).

[12] P. M. Smith and D. A. L. Paul, Can. J. Phys. 48, 2984 (1970).

[13] G. K. Ivanov, Dokl. Akad. Nauk SSSR 291622 (1986); Chem. Phys. Lett. 135, 89 (1987).

[14] G. Laricchia and C. Wilkin, Phys. Rev. Lett. 79, 2241 (1997)

[15] F. A. Gianturco, T. Mukherjee and A. Occhigrossi, Phys. Rev. A 64, 032715 (2001); A. Occhigrossi and F. A. Gianturco, J. Phys. B 36, 1383 (2003); J. Franz and F. A. Gianturco, Nucl. Instrum. Methods B 247, 20 (2006).

[16] E. P. da Silva, J. S. E. Germano, and M. A. P. Lima, Phys. Rev. A 49, R1527 (1994); M. T. do N. Varella, C. R. C. de Carvalho, M. A. P. Lima, Nucl. Instrum. Methods B 192, 225 (2002).

[17] T. Nishimura and F. A. Gianturco, Phys. Rev. Lett. 90, 183201 (2003); Phys. Rev. A 72, 022706 (2005).

[18] G. F. Gribakin, Phys. Rev. A 61, 022720 (2000).

[19] G. F. Gribakin, in New Directions in Antimatter Chemistry and Physics, Eds. C. M. Surko and F. A. Gianturco (Kluwer Academic Publishers, Netherlands, 2001), p. 413.

[20] G. F. Gribakin and P. M. W. Gill, Nucl. Instrum. and Methods B 221, 30 (2004).

[21] S. J. Gilbert et al. Phys. Rev. Lett. 88, 043201 (2002).

[22] L. D. Barnes, S. J. Gilbert, and C. M. Surko, Phys. Rev. A 67, 032706 (2003).

[23] J. Mitroy, M. W. J. Bromley, and G. G. Ryzhikh, J. Phys. B 35, R81 (2002); H. Chojnacki and K. Strasburger, Mol. Phys. 104, 2273 (2006) and Refs. therein.

[24] L. D. Landau and E. M. Lifshitz, Quantum Mechanics, 3rd ed. (Pergamon, Oxford, 1977).

[25] Yu. N. Demkov and V. N. Ostrovsky, Zero-Range Potentials and their Applications in Atomic Physics (Plenum Press, New York, 1988).

[26] N. F. Lane, Rev. Mod. Phys. 52, 29 (1980).

[27] J. P. Marler and C. M. Surko, Phys. Rev. A, 72, 062702 (2005); J. P. Marler, G. F. Gribakin and C. M. Surko, Nucl. Instrum. Methods B 247, 87 (2006).

[28] ${ }_{2} F_{1}\left(\frac{1}{2}, 1 ; \frac{5}{2} ;-z^{2}\right)=\frac{3}{2} z^{-2}\left[\left(1+z^{2}\right) z^{-1} \arctan z-1\right]$.

[29] L. M. Bishop and L. M. Cheung, J. Phys. Chem. Ref. 
Data, 11, 119 (1982).

[30] V. A. Dzuba et al. Phys. Scripta T46, 248, (1993); J. Phys. B 29, 3151 (1996).
[31] G. M. Stewart and J. D. McDonald, J. Chem. Phys. 78, 3907 (1983). 